Research Paper

\title{
Left ventricular diastolic and systolic dyssynchrony and dysfunction in heart failure with preserved ejection fraction and a narrow QRS complex
}

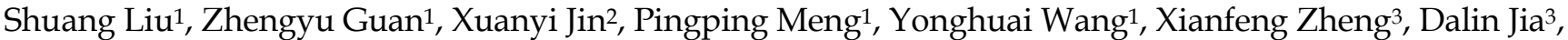
Chunyan Ma1 ${ }^{\boxplus}$, Jun Yang${ }^{1}$

1. Department of Cardiovascular Ultrasound, The First Hospital of China Medical University, Shenyang, Liaoning, People's Republic of China, 110001;

2. Department of Cardiology, Mayo Clinic (Arizona), Scottsdale, Arizona, United States, 85259;

3. Department of Cardiology, The First Hospital of China Medical University, Shenyang, Liaoning, People's Republic of China.

$\triangle$ Corresponding author: Chunyan Ma, Address: The First Hospital of China Medical University, 155 Nanjing Bei Street, Heping District, Shenyang, Liaoning 110001, China Fax: +86 248328 2114; Telephone: +86-13998816448; Email: cmu1h_mcy@126.com

(C) Ivyspring International Publisher. This is an open access article distributed under the terms of the Creative Commons Attribution (CC BY-NC) license (https://creativecommons.org/licenses/by-nc/4.0/). See http://ivyspring.com/terms for full terms and conditions.

Received: 2017.07.17; Accepted: 2017.10.12; Published: 2018.01.01

\begin{abstract}
Aims: Mechanical dyssynchrony has been reported in heart failure with preserved ejection fraction (HFpEF), with a majority of patients having a narrow QRS complex; however, whether any benefit is observed with restoration of dyssynchrony remains unclear. We sought to assess left ventricular (LV) dyssynchrony and function in HFpEF and elucidate the underlying mechanisms that may account for HFpEF.

Methods: Seventy-eighty patients with a narrow QRS complex including 47 with HFpEF, 31 with heart failure with reduced ejection fraction (HFrEF) patients, and 29 with asymptomatic left ventricular diastolic dysfunction (LVDD) were recruited. Forty-five normal subjects acted as controls. Systolic LV longitudinal strain (LS), systolic longitudinal strain rate (LSrS), early diastolic longitudinal strain rate (LSrE), and late diastolic longitudinal strain rate (LSrA) were measured using speckle tracking echocardiography. LV diastolic and systolic dyssynchrony (Te-SD and Ts-SD) were calculated.

Results: Te-SD and Ts-SD were prolonged in HFpEF and HFrEF patients than in the control group $(p<0.05)$. However, Ts-SD was shorter in HFpEF patients compared to HFrEF patients despite a narrow QRS complex $(p<0.05)$. LV global LS, LSrS, and LSrE were decreased in patients with HFpEF and HFrEF compared to other groups, with HFrEF being even more reduced than HFpEF $(p<0.05)$. Reduced LS, LSrS, and LSrE could effectively differentiate HF from asymptomatic LVDD patients $(p<0.05)$.

Conclusion: HFrEF exhibited increased systolic dyssynchrony compared to HFpEF despite a narrow QRS complex in addition to the more reduced diastolic and systolic function. Therefore, targeting to improve diastolic and systolic function instead of managing systolic dyssynchrony might be of great importance in the treatment of HFpEF.
\end{abstract}

Key words: Dyssynchrony, Heart failure with preserved ejection fraction, Narrow QRS complex, Speckle tracking echocardiography.

\section{Introduction}

Heart failure with preserved ejection fraction (HFpEF) now accounts for approximately half of chronic heart failure (HF) patients and carries a dismal prognosis [1]. Multiple complex pathophysiological mechanisms have been described and, clinically, many patients will present with a narrow QRS complex [2]. Left ventricular diastolic dysfunction (LVDD) has long been considered as the main cause of HFpEF, however, large previous clinical trials failed to improve the prognosis of HFpEF by restoring LV diastolic function $[3,4,5]$. Therefore, new pathophysiologic paradigms with the goal of developing novel therapeutic regimens in HFpEF arose. 
A previous study examined the importance of mechanical dyssynchrony in the development of HFpEF, which suggested that restoration of systolic dyssynchrony could help to improve the symptomatology of patients with HFpEF [6]. However, little evidence exists to demonstrate that cardiac-resynchronization therapy (CRT) benefits patients with HFpEF, despite a study showing a clinical and structural improvement in patients with a mean left ejection fraction (LVEF) of $43 \pm 7 \%$ after CRT [7]. In addition, although mechanical dyssynchrony exists in about $30 \%$ to $40 \%$ of heart failure with reduced ejection fraction (HFrEF) patients with a narrow QRS duration [8, 9], the large multicenter randomized controlled clinical trial, EchoCRT, failed to conclude that CRT benefits HF patients with mechanical dyssynchrony without QRS widening [10]. Still, the indication of CRT in HFpEF patients remains controversial $[2,11,12]$.

Speckle tracking echocardiography (STE) is a robust assessment tool of mechanical dyssynchrony derived from the regional timing of contraction and relaxation of the myocardium $[1,12]$. In the present study, we hypothesized that LV systolic dyssynchrony accounted for the underlying mechanisms of HFpEF, which may provide further insight into the understanding of this complex disorder and spearhead the exploration of more patient-specific therapeutic strategies. For this, we comprehensively assessed the mechanical dyssynchrony and function in HFpEF with a narrow QRS by STE and compared these to patients with HFrEF with a narrow QRS, asymptomatic LVDD patients, and normal healthy subjects with the aim of validating the hypothesis.

\section{Methods}

\section{Patient selection}

A total of 107 patients including 29 asymptomatic patients (14 males and 15 females), 47 patients with HFpEF (20 males and 27 females) and 31 patients with HFrEF (17 males and 14 females) were included for this study conducted in the First Hospital of China Medical University (Shenyang, Liaoning Province, China). HF was diagnosed according to the current recommendations [13], and LVDD was distinguished according to the latest American Society of Echocardiography (ASE) criteria [14]. The HFpEF group had a LVEF $>50 \%$ while the HFrEF group had a LVEF $<50 \%$ [14]. Patients with rhythms other than sinus and those with a QRS duration of $>130 \mathrm{~ms}$, in addition to those with valvular heart disease, cardiomyopathy, severe pulmonary disease, constrictive pericarditis, LV systolic dysfunction, other associated systemic diseases, and poor echocardiographic views were excluded from this study.

Forty-five healthy volunteers (22 males and 23 females) comprising of medical students and members of the local community with no history of cardiovascular or systemic diseases, abnormal echocardiographic findings, or HF symptoms were enrolled as normal controls (control group). The study protocol was approved by the ethics committee of China Medical University and written informed consent was obtained from all participants.

\section{Echocardiography}

Standard echocardiography with Doppler studies was performed using a Vivid 7 Dimension ultrasound system (GE Healthcare, Waukesha, WI, USA) equipped with a 2-4 MHz phased array probe. All images and measurements were acquired from standard views, and digitally stored for offline analysis. LV diameters, volumes, mass of hypertrophic LV, LVEF, LA volume, and LV diastolic function were measured in accordance with the ASE guidelines[15] The left atrial diameter (LAD), LV end-diastolic and systolic dimension (LVEDD and LVESD), interventricular septal and posterior wall thicknesses (IVSD and PWD), and LV mass index (LVMI) were measured and calculated. The LVEF was measured using the biplane modified Simpson's method. Peak early (E) and late (A) diastolic velocities across the mitral valve were measured, and the E/A ratio were calculated. The peak early diastolic mitral annular velocity $\left(\mathrm{e}^{\prime}\right)$ was measured at the levels of the mitral septal annulus ( $\left.\mathrm{e}_{\text {sep }}^{\prime}\right)$ and lateral annulus ( $\mathrm{e}^{\prime}$ lat) with an apical four-chamber view, and the E/e' ratio was calculated. The $\mathrm{LV}$ end-diastolic pressure $\left(\right.$ LVEDP $\left._{\text {echo }}\right)$ was estimated at $11.96+0.596{ }^{*} \mathrm{E} / \mathrm{e}^{\prime}[16]$.

\section{STE data collection}

For LV strain and strain rate analysis, dynamic two-dimensional ultrasound images of three cardiac cycles from long-axis, apical four-chamber, and two-chamber views were acquired at a frame rate of 57-72 frames per second. The images were analyzed using customized software with the EchoPAC work station (GE Healthcare). The endocardial LV boundary was delineated manually and then the software automatically drew the epicardial boundary. The widths of the regions of interest were manually adjusted to match the actual endocardial and epicardial boundaries. An automatically generated region of interest was divided into six segments. The LV peak longitudinal systolic strain (LS), LV peak LS rate (LSrS), early diastolic strain rate (LSrE), and late diastolic strain rate (LSrA) were calculated. The final 
strain parameters were the averages of the values obtained from the three apical views. The times to LS (Ts) and LSre (Te) of every segment were measured with reference to the QRS complex. LV systolic and diastolic dyssynchrony were calculated as the standard deviations of the Td (Te-SD) and Ts (Ts-SD) values of all LV segments $[17,18]$.

\section{Statistical analysis}

Statistical analysis was performed using SPSS version 17.0 software (SPSS Inc., Chicago, IL, USA). Descriptive data are summarized as the percentage frequency for categorical variables and the mean \pm standard deviation (SD) for continuous variables. Continuous variables between two groups were analyzed using the unpaired Student's $t$-test or Mann-Whitney U-test, and categorical data were analyzed using the Fisher exact test or chi-squared test, as appropriate. Differences between multiple groups were compared using one-way analysis of variance with LSD correction for the least significant difference. The Pearson coefficient was used for correlation analysis. Receiver-operating characteristic (ROC) curve analysis was employed to identify parameters that were best associated with HF symptoms. Optimal cut-off values were selected at the highest sum of sensitivity and specificity. A two-tailed probability $(p)$ value $<0.05$ was considered statistically significant. intra- and inter- observer variability was determined by calculating the coefficients of variation, which were calculated as the standard deviations of differences between repeated measurements divided by the average value of those measurements and expressed as percentages.

\section{Results}

\section{Clinical characteristics}

The comorbidities of patients with HFpEF and asymptomatic LVDD were characterized by the presence of type 2 diabetes, hypertension, history of coronary heart disease and obesity, while HFrEF patients had a higher prevalence of dilated cardiomyopathy and coronary heart disease. The plasma N-terminal pro B-type natriuretic peptide (NT-pro BNP) level was significantly higher in patients with HFpEF than that in LVDD patients. Moreover, many patients with HFrEF had higher New York Heart Association functional class than those with HFpEF (Table 1).

The LVEF was significantly decreased in HFrEF group compared to other groups $(p<0.05)$, however, LVEF was not statistically significant among normal control, asymptomatic LVDD patients, and the HFpEF groups. LVEDD, IVSD, PWD, and LAD values were significantly increased in patients with LVDD and HFpEF than in normal controls $(p<0.05)$. Moreover, the LVEDP echo and E/ $\mathrm{e}^{\prime}$ values in patients with asymptomatic LVDD, HFpEF, and HFrEF were significantly increased, while $\mathrm{e}^{\prime}$ of the mitral annular velocity was significantly decreased, than that of the control group $(p<0.05)$. Although the differences in

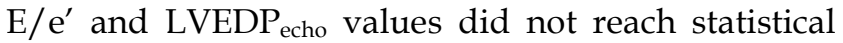
significance, they tended to be higher in patients with HFpEF than those with asymptomatic LVDD, moreover, the E/e' and LVEDP ${ }_{\text {echo }}$ were significantly higher than the other three groups (Table 2).

Table 1. Comparison of clinic characteristics of HF patients with study controls

\begin{tabular}{|c|c|c|c|c|}
\hline $\begin{array}{l}\text { Comparison of clinic } \\
\text { characteristics }\end{array}$ & $\begin{array}{l}\text { Control } \\
(\mathrm{n}=45)\end{array}$ & $\begin{array}{l}\text { LVDD } \\
(\mathrm{n}=29)\end{array}$ & $\begin{array}{l}\text { HFpEF } \\
(\mathrm{n}=47)\end{array}$ & $\operatorname{HFrEF}(n=31)$ \\
\hline Age (years) & $58 \pm 13$ & $62 \pm 8$ & $61 \pm 13$ & $63 \pm 15$ \\
\hline QRS duration (ms) & $92 \pm 13$ & $91 \pm 10$ & $93 \pm 11$ & $99 \pm 17$ \\
\hline Hypertension (n) & & $27(93 \%)$ & $36(82 \%)$ & $22(71 \%)$ \\
\hline History of CAD (n) & & $9(31 \%)$ & $18(41 \%)$ & $14(45 \%)$ \\
\hline Type 2 Diabetes mellitus (n) & & $12(41 \%)$ & $17(39 \%)$ & $11(35 \%)$ \\
\hline Obesity (n) & & $6(21 \%)$ & $7(16 \%)$ & $9(29 \%)$ \\
\hline Dilated Cardiomyopathy & & & & $19(61 \%)$ \\
\hline \multicolumn{5}{|l|}{ NYHA classification n (\%) } \\
\hline II & & & $29(62 \%)$ & $4(13 \%)$ \\
\hline III & & & $17(36 \%)$ & $18(58 \%)$ \\
\hline IV & & & $1(2 \%)$ & $9(29 \%)$ \\
\hline NT pro-BNP $(\mathrm{pg} / \mathrm{ml})$ & & $32 \pm 16$ & $492 \pm 501^{\#}$ & $1240 \pm 1246^{\# \&}$ \\
\hline
\end{tabular}

\section{LV function and dyssynchrony}

The LV function and dyssynchrony values are summarized in Table 3. The LV global LS, LSrS, and LSrE values were significantly decreased in patients with HFpEF than in normal controls and asymptomatic LVDD patients, which were even more decreased in HFrEF patients $(p<0.05)$. However, there was no difference in global LS and LSrS values in asymptomatic LVDD patients compared to normal controls. Although Te-SD and Ts-SD were significantly more prolonged in the HFpEF and the HFrEF groups than in the control group $(p<0.05)$, however, Ts-SD was shorter in the HFpEF group than the HFrEF group (Figure 1).

According to the ROC curve analysis, LV global LS, LSrS, and LSrE could efficiently differentiate HF symptoms from asymptomatic LVDD patients. LV global LSrE with a cut-off value of 0.95 had the highest AUC (sensitivity, 83.1\%; specificity, 87.5\%; area under the curve $=0.929 ; 95 \%$ confidence interval $[\mathrm{CI}]=0.870-0.987 ; p<0.001)($ Figure 2$)$. 
Table 2. Comparison of conventional echocardiography of HF patients versus study controls

\begin{tabular}{|c|c|c|c|c|}
\hline & Control $(n=45)$ & $\operatorname{LVDD}(n=29)$ & HFpEF (n=47) & HFrEF $(n=31)$ \\
\hline LVEDD(mm) & $47.40 \pm 3.26$ & $51.60 \pm 3.76^{*}$ & $52.66 \pm 6.76^{*}$ & $67.63 \pm 9.50^{* \# \& ~}$ \\
\hline $\operatorname{LVESD}(\mathrm{mm})$ & $32.09 \pm 3.15$ & $36.30 \pm 3.80$ & $37.98 \pm 7.64^{*}$ & $59.30 \pm 10.92^{\text {*\#\& }}$ \\
\hline IVS (mm) & $7.60 \pm 0.79$ & $9.17 \pm 1.29^{*}$ & $9.30 \pm 1.98^{*}$ & $8.41 \pm 1.45$ \\
\hline PW (mm) & $7.54 \pm 0.76$ & $8.60 \pm 0.81^{*}$ & $8.98 \pm 2.03^{*}$ & $8.23 \pm 1.34$ \\
\hline LVMI (g/m2) & $67.51 \pm 12.14$ & $86.87 \pm 17.06$ & $100.95 \pm 28.53^{*}$ & $132.15 \pm 45.84^{* \# \& ~}$ \\
\hline LAD (mm) & $33.16 \pm 4.29$ & $40.93 \pm 5.33^{*}$ & $41.00 \pm 4.64^{*}$ & $46.43 \pm 7.65^{\text {\#\#\& }}$ \\
\hline LVEF (\%) & $63.07 \pm 4.89$ & $61.93 \pm 3.98$ & $59.72 \pm 6.23^{*}$ & $31.20 \pm 8.02^{* \# \& \&}$ \\
\hline Mitral E/A & $1.29 \pm 0.42$ & $0.86 \pm 0.27$ & $1.04 \pm 0.73$ & $1.89 \pm 1.20^{* \# \&}$ \\
\hline $\mathrm{e}^{\prime}{ }_{\text {sep }}(\mathrm{cm} / \mathrm{s})$ & $9.13 \pm 2.18$ & $6.32 \pm 1.83^{*}$ & $5.59 \pm 1.42^{*}$ & $3.69 \pm 1.37^{* \# \&}$ \\
\hline $\mathrm{e}^{\prime}$ lat $(\mathrm{cm} / \mathrm{s})$ & $12.68 \pm 3.42$ & $8.00 \pm 2.36^{*}$ & $7.59 \pm 2.28^{*}$ & $5.60 \pm 2.77^{* \# \&}$ \\
\hline Mitral E/ e' & $7.64 \pm 1.87$ & $10.78 \pm 2.60$ & $12.10 \pm 4.95^{*}$ & $22.09 \pm 10.29^{\text {*\#\& }}$ \\
\hline LVEDP $_{\text {echo }}$ & $16.52 \pm 1.11$ & $18.39 \pm 1.55$ & $19.17 \pm 2.95^{*}$ & $25.13 \pm 6.13^{\text {*\#\& }}$ \\
\hline
\end{tabular}

${ }^{*} P<0.05$ versus control group, ${ }^{*} P<0.05$ versus $L V D D, \& P<0.05$ versus HFpEF. LVDD, left ventricular diastolic dysfunction; HFpEF, heart failure with preserved ejection fraction; HFrEF, heart failure with reduced ejection fraction; LVEDD, left ventricular end-diastolic diameter; LVESD, left ventricular end-systolic diameter; LVMI, left ventricular mass index; LAD, left atrium diameter; LVEF, left ventricular ejection fraction; LVEDP, left ventricular end diastolic pressure.

Table 3. Comparison of left ventricular function and dyssynchrony between groups

\begin{tabular}{|c|c|c|c|c|}
\hline & Control $(n=45)$ & LVDD (n=29) & HFpEF $(n=47)$ & $\operatorname{HFrEF}(n=31)$ \\
\hline Te-SD (ms) & $23 \pm 7$ & $31 \pm 14$ & $38 \pm 15^{*}$ & $39 \pm 16^{*}$ \\
\hline Ts-SD (ms) & $33 \pm 12$ & $49 \pm 16^{*}$ & $55 \pm 13^{*}$ & $65 \pm 19^{* \# \&}$ \\
\hline Global S (\%) & $-19.94 \pm 2.35$ & $-18.48 \pm 2.98$ & $-15.53 \pm 3.19^{* \#}$ & $-8.82 \pm 1.95^{\star \# \& ~}$ \\
\hline Global SRs (1/s) & $-1.13 \pm 0.18$ & $-1.06 \pm 0.16$ & $-0.79 \pm 0.20^{* \#}$ & $-0.46 \pm 0.13^{* \# \&}$ \\
\hline Global SRe (1/s) & $1.56 \pm 0.32$ & $1.19 \pm 0.27^{*}$ & $0.75 \pm 0.24^{* \#}$ & $0.46 \pm 0.15^{\star \# \& ~}$ \\
\hline Global Sra (1/s) & $0.96 \pm 0.20$ & $1.09 \pm 0.22^{*}$ & $0.84 \pm 0.28^{\#}$ & $0.43 \pm 0.26^{* \# \&}$ \\
\hline
\end{tabular}

${ }^{*} P<0.05$ versus control group, $\# P<0.05$ versus $L V D D, \& P<0.05$ versus HFpEF. LVDD, left ventricular diastolic dysfunction; $H F p E F$, heart failure with preserved ejection fraction; HFrEF, heart failure with reduced ejection fraction; Te-SD, standard deviation of time to peak early diastolic strain rate; Ts-SD, standard deviation of time to peak systolic strain.
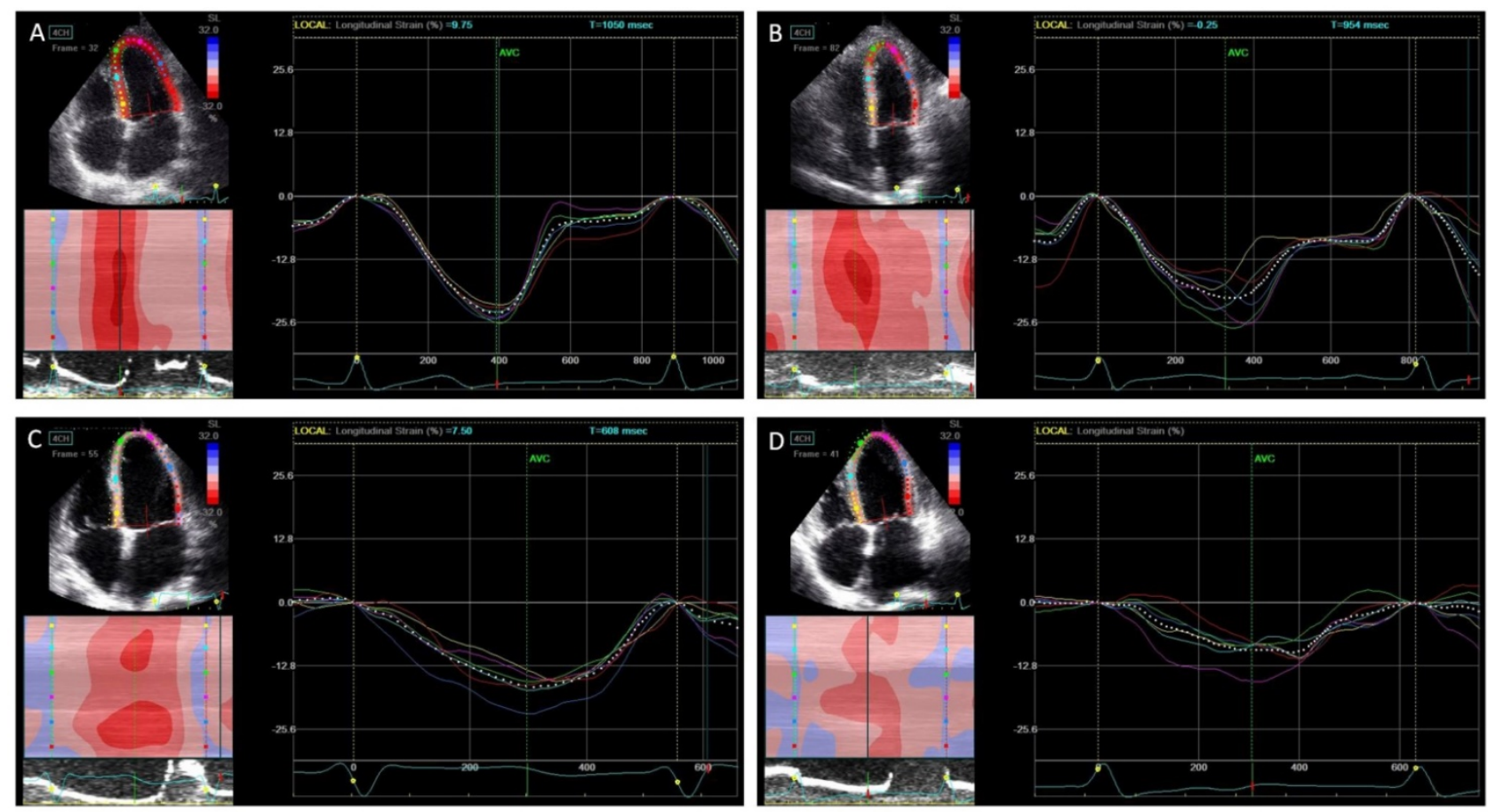

Figure 1. Peak systolic longitudinal strain and dyssynchrony. In normal controls (A), asymptomatic left ventricular diastolic dysfunction (LVDD) patients (B), heart failure with preserved ejection fraction (HFpEF) patients (C), and heart failure with reduced ejection fraction (HFrEF) (D). The peak longitudinal strain was decreased gradually from each group while the systolic dyssynchrony was increased.

\section{Correlation Analysis}

LS was negatively correlated with Te-SD $(r=-0.382, p<0.001)$ and Ts-SD $(r=-0.523, p<0.001)$, and positively LVEF $(r=0.817, p<0.001)$. Moreover, $\mathrm{e}^{\prime}$ lat was negatively correlated with Te-SD $(r=-0.405$, $p<0.001)$ and positively correlated with LSrE $(r=0.766$, $p<0.001)$. Furthermore, LSrE was negatively correlated with Te-SD and Ts-SD $(r=-0.622$ and 
-0.541 , respectively, $p<0.001$ ) in all participants. However, we didn't find any correlation between dyssynchrony and the width of the QRS complex.

\section{Reproducibility}

Twenty patients were randomly selected for repeat measurements. The intra- and inter-observer coefficients of variation were $5.4 \%$ and $7.1 \%$ for the strain and strain rate, respectively. The coefficients of variation for intra- and inter-observer variability were $7.9 \%$ and $9.2 \%$ for dyssynchrony parameters, respectively.

\section{Discussion}

The major findings of the present study were as follows: 1) LV diastolic and systolic synchronies were significantly prolonged in both HFpEF and HFrEF with a narrow QRS complex patients than in the control group, however, the systolic dyssynchrony was shortened in HFpEF compared to that in HFrEF with a narrow QRS duration, although diastolic dyssynchrony didn't reach statistical significance between the two groups; 2) LV longitudinal systolic function was significantly decreased in HFpEF with a narrow QRS than in asymptomatic LVDD patients and normal controls; it was even more reduced in HFrEF with a narrow QRS patients; 3) reduced LV diastolic and systolic function could efficiently differentiate patients with or without HF (preserved and reduced $\mathrm{EF}$ ).

HFpEF accounts for approximately $50 \%$ of all HF patients, which is characterized by the presence of LVDD evident from slow LV relaxation and increased LV stiffness [19]. However, restoring LV diastolic function failed to improve the prognosis of HFpEF as previously mentioned $[3,4,5]$. Moreover, LVDD is not unique to patients with HFpEF; previous studies reported that LVDD also occurred in HFrEF, and correlated well with symptoms than LVEF [20, 21]. Therefore, the underlying pathophysiology of HFpEF is still debated despite diverse mechanisms including pulmonary hypertension, reduced peripheral oxygen utilization, and increased arterial stiffness [1]. Additionally, there is no evidence-based management for improving mortality in HFpEF patients.

Mechanical dyssynchrony is a term used to describe systolic and diastolic mechanical variability. A previous study has suggested that approximately $30 \%$ of patients with a narrow QRS have mechanical dyssynchrony [22]. Dyssynchronous contraction is followed by the synchronous electrical activation in the LV preventing normal myocardial activation and contraction [8]. Regional heterogeneity in LV contraction is due to the small heterogeneous areas of myocardial fibrosis that may produce dyssynchronous contraction without causing an electrical impact on QRS morphology [8].

The majority of HFpEF patients have a narrow QRS, although diastolic and systolic dyssynchronies are very common [2]. In the present study, we found the diastolic and systolic dyssynchronies in the HFpEF and the HFrEF groups were significantly increased compared to normal subjects despite the narrow QRS complex, however, we didn't find any correlation between the width of QRS and dyssynchrony, indicating that electromechanical coupling delay is not a major factor for the observed LV dyssynchrony. The underlying causes of HFpEF, including hypertension, type 2 diabetes mellitus, and coronary artery disease, which first damage the most susceptible subendocardial myocardial fibers [23], may account for the increased mechanical dyssynchrony in HFpEF patients as we demonstrated in this study.

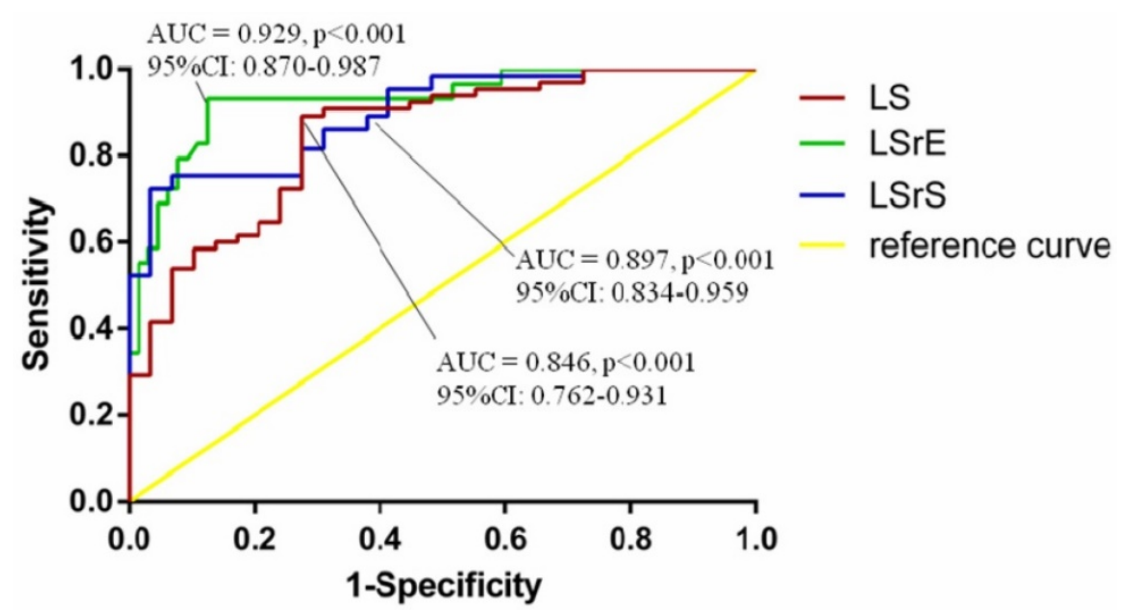

Figure 2. Receiver-operating characteristic curve analyses of echocardiographic parameters for diagnosis of heart failure. AUC, area under the curve; Cl, confidence interval; LS, longitudinal strain; LSrE, early diastolic longitudinal strain rate; LSrS, systolic longitudinal strain rate. 
Biventricular pacing was proposed as an effective treatment for HF with prolonged QRS duration, which could improve symptoms, LV function, and mortality. However, there is no evidence of benefit in patients with HFrEF with a narrow QRS duration [24]. Furthermore, CRT did not improve the quality of life or peak oxygen consumption in patients with a narrow QRS duration and evidence of echocardiographic dyssynchrony in a large and randomized clinical trial [25]. A previous study found LV dyssynchrony was prolonged in HFpEF and proposed that restoration of LV dyssynchrony could be the new therapeutic pathway for HFpEF [6]. However, in the present study, although the LV systolic dyssynchrony was prolonged in HFpEF patients, it was still lower than HFrEF with a narrow QRS. In this regard, we consider CRT might not be a good option for HFpEF with a narrow QRS.

The prolonged diastolic and systolic dyssynchronies indicate energy wastage resulting from LV dyssynchrony, which may lead to a reduction in cardiac energy reserves [11]. Moreover, a reduction in systolic shortening resulting from deteriorated dyssynchrony has been shown [26]. Despite a more decreased LV longitudinal systolic function, a more prolonged systolic dyssynchrony was observed in HFrEF patients compared to HFpEF patients in the present study. Additionally, we also found that LV systolic function was significantly correlated with LV diastolic and systolic dyssynchrony, indicating an underlying relationship between LV dysfunction and increased dyssynchrony in HFpEF and HFrEF.

Diastolic dysfunction has long been considered as a key pathophysiologic mediator of HFpEF; the characteristics of concomitant systolic dysfunction has not been well defined, although longitudinal dysfunction resulting from comorbidities such as diabetes, coronary artery disease and hypertension have been shown to play an important role in patients with HFpEF [27]. Physiological studies also suggested that mechanical dyssynchrony impairs LV ejection efficiency $[10,28]$. In the present study, apart from the prolonged diastolic and systolic dyssynchrony in HFpEF and HFrEF, a decreased LV longitudinal diastolic and systolic dysfunction was observed in those groups, and LV dyssynchronies correlated well with LV dysfunction. Therefore, LV dyssynchronies may be partly responsible for the LV dysfunction. Moreover, global LS, LSrS, and LSrE could efficiently differentiate HF symptoms from asymptomatic LVDD patients, indicating the LV dysfunction potentially contribute to the presence of $\mathrm{HF}$ symptoms. Therefore, treatment destined to improve
LV diastolic and systolic function might be of great importance in the treatment of HFpEF to prevent the occurrence of HFrEF.

\section{Study limitations}

The major limitation of this study was the lack of a prospective evaluation to assess the prognostic differences between asymptomatic LVDD, HFpEF, and HFrEF. Long-term follow-up is needed to verify the prognostic value of LV dysfunction and dyssynchrony in HFpEF. Moreover, we only included HFpEF with a narrow QRS complex because the majority of our patients had a narrow QRS; further research should focus on the differences between HFpEF with both narrow and wide QRS complexes. Furthermore, the sample size was relatively small because it was difficult to recruit a sufficient number of HF patients from a single hospital. Hence, further multicenter studies with larger numbers of patients are needed to validate these findings.

\section{Conclusions}

In this study, we found the systolic dyssynchrony was shorter in patients with HFpEF than in HFrEF with narrow QRS, suggesting that resynchronization might not be a suitable management option for such patients. Moreover, the LV systolic function was significantly reduced in patients with HFpEF and HFrEF with a narrow QRS, and decreased LV diastolic and systolic function could effectively differentiate HF from asymptomatic LVDD patients. Therefore, management with the goal of improving LV diastolic and systolic function instead of resynchronization may be considered a possible therapeutic pathway for HFpEF.

\section{Abbreviations}

LV: left ventricular; LVDD: left ventricular diastolic dysfunction; HFpEF: heart failure with preserved ejection fraction; LS: longitudinal strain; LSrS: systolic longitudinal strain rate; LSrE: early diastolic longitudinal strain rate; LSrA: late diastolic longitudinal strain rate; Te-SD: LV diastolic dyssynchrony; Ts-SD: LV systolic dyssynchrony; LVEDD: LV end-diastolic dimension; HF: heart failure; HFrEF: HF with reduced ejection fraction; STE: Speckle tracking echocardiography; ASE: American Society of Echocardiography; LAD: left atrial diameter; LVEDD: LV end-diastolic dimension; LVESD: LV end-systolic dimension; IVSD: interventricular septal thicknesses; PWD: posterior wall thicknesses; LVMI: LV mass index; E: Peak early; A: Peak late; LVEDP echo: LV end-diastolic pressure; Ts: times to LS; Te: times LSre; SD: standard deviation; ROC: Receiver-operating characteristic; 
NT-pro BNP: N-terminal pro B-type natriuretic peptide; CI: confidence interval.

\section{Acknowledgements}

The study was supported by National Natural Science Foundation of China (NO. 81401413) and Scientific Research of The First Hospital of China Medical University (Number:fsfh1312).

\section{Competing Interests}

The authors have declared that no competing interest exists.

\section{References}

1. Santos AB, Kraigher-Krainer E, Bello N, et al. Left ventricular dyssynchrony in patients with heart failure and preserved ejection fraction. European Heart Journal. 2014; 35: 42-7.

2. Yu CM, Zhang Q, Yip GW, et al. Diastolic and systolic asynchrony in patients with diastolic heart failure: a common but ignored condition. J Am Coll Cardiol. 2007; 49: 97-105.

3. Yusuf S, Pfeffer MA, Swedberg K, et al. Effects of candesartan in patients with chronic heart failure and preserved left-ventricular ejection fraction: the CHARM-Preserved Trial. Lancet. 2003; 362: 777-81.

4. Cleland JG, Tendera M, Adamus J, et al. The perindopril in elderly people with chronic heart failure (PEP-CHF) study. European Heart Journal. 2006; 27: 2338-45.

5. Massie BM, Carson PE, McMurray JJ, et al. Irbesartan in patients with heart failure and preserved ejection fraction. N Engl J Med. 2008; 359: 2456-67.

6. Morris DA, Vaz Perez A, Blaschke F, et al. Myocardial systolic and diastolic consequences of left ventricular mechanical dyssynchrony in heart failure with normal left ventricular ejection fraction. Eur Heart J Cardiovasc Imaging. 2012; 13: 556-67.

7. Chung ES, Katra RP, Ghio $S$, et al. Cardiac resynchronization therapy may benefit patients with left ventricular ejection fraction $>35 \%$ : a PROSPECT trial substudy. Eur J Heart Fail. 2010; 12: 581-7.

8. Jackson T, Claridge S, Behar J, et al. Narrow QRS systolic heart failure: is there a target for cardiac resynchronization? Expert Rev Cardiovasc Ther. 2015; 13: 783-97.

9. Marechaux S, Menet A, Guyomar Y, et al. Role of echocardiography before cardiac resynchronization therapy: new advances and current developments. Echocardiography. 2016; 33: 1745-52.

10. Gorcsan J, 3rd, Sogaard P, Bax JJ, et al. Association of persistent or worsened echocardiographic dyssynchrony with unfavourable clinical outcomes in heart failure patients with narrow QRS width: a subgroup analysis of the EchoCRT trial. European Heart Journal. 2016; 37: 49-59.

11. Phan TT, Abozguia K, Shivu GN, et al. Myocardial contractile inefficiency and dyssynchrony in heart failure with preserved ejection fraction and narrow QRS complex. J Am Soc Echocardiogr. 2010; 23: 201-6.

12. Biering-Sorensen T, Shah SJ, Anand I, et al. Prognostic importance of left ventricular mechanical dyssynchrony in heart failure with preserved ejection fraction. Eur J Heart Fail; in press.

13. Ponikowski P, Voors AA, Anker SD, et al. 2016 ESC Guidelines for the diagnosis and treatment of acute and chronic heart failure: The Task Force for the diagnosis and treatment of acute and chronic heart failure of the European Society of Cardiology (ESC)Developed with the special contribution of the Heart Failure Association (HFA) of the ESC. European Heart Journal. 2016; 37: 2129-200

14. Nagueh SF, Smiseth $\mathrm{OA}$, Appleton $\mathrm{CP}$, et al. Recommendations for the Evaluation of Left Ventricular Diastolic Function by Echocardiography: An Update from the American Society of Echocardiography and the European Association of Cardiovascular Imaging. J Am Soc Echocardiogr. 2016; 29: 277-314

15. Lang RM, Badano LP, Mor-Avi V, et al. Recommendations for cardiac chamber quantification by echocardiography in adults: an update from the American Society of Echocardiography and the European Association of Cardiovascular Imaging. Eur Heart J Cardiovasc Imaging. 2015; 16: 233-70.

16. Nagueh SF. Echocardiographic assessment of left ventricular relaxation and cardiac filling pressures. Curr Heart Fail Rep. 2009; 6: 154-9.

17. Leong DP, Hoogslag GE, Piers SR, et al. The relationship between time from myocardial infarction, left ventricular dyssynchrony, and the risk for ventricular arrhythmia: speckle-tracking echocardiographic analysis. J Am Soc Echocardiogr. 2015; 28: 470-7.

18. Sun JP, Xu TY, Lee AP, et al. Early diastolic dyssynchrony in relation to left ventricular remodeling and function in hypertension. Int J Cardiol. 2015; 179: $195-200$

19. Wang CL, Powell BD, Redfield MM, et al. Left ventricular discoordination index measured by speckle tracking strain rate imaging predicts reverse remodelling and survival after cardiac resynchronization therapy. Eur J Heart Fail. 2012; 14: 517-25

20. Hadano $Y$, Murata $\mathrm{K}$, Yamamoto $\mathrm{T}$, et al. Usefulness of mitral annular velocity in predicting exercise tolerance in patients with impaired left ventricular systolic function. Am J Cardiol. 2006; 97: 1025-8.

21. Skaluba SJ, Litwin SE. Mechanisms of exercise intolerance: insights from tissue Doppler imaging. Circulation. 2004; 109: 972-7.

22. Foley PW, Khadjooi K, Ward JA, et al. Radial dyssynchrony assessed by cardiovascular magnetic resonance in relation to left ventricular function, myocardial scarring and QRS duration in patients with heart failure. J Cardiovasc Magn Reson. 2009; 11: 50.

23. Szelenyi Z, Fazakas A, Szenasi G, et al. The mechanism of reduced longitudinal left ventricular systolic function in hypertensive patients with normal ejection fraction. J Hypertens. 2015; 33: 1962-69; discussion 1969.

24. Brignole M, Auricchio A, Baron-Esquivias G, et al. 2013 ESC Guidelines on cardiac pacing and cardiac resynchronization therapy. Rev Esp Cardiol (Engl Ed). 2014; 67: 58

25. Beshai JF, Grimm RA, Nagueh SF, et al. Cardiac-resynchronization therapy in heart failure with narrow QRS complexes. N Engl J Med. 2007; 357: 2461-71.

26. Kuznetsova $\mathrm{T}$, Bogaert $\mathrm{P}$, Kloch-Badelek M, et al. Association of left ventricular diastolic function with systolic dyssynchrony: a population study. Eur Heart J Cardiovasc Imaging. 2013; 14: 471-9.

27. Morris DA, Boldt LH, Eichstadt H, et al. Myocardial systolic and diastolic performance derived by 2-dimensional speckle tracking echocardiography in heart failure with normal left ventricular ejection fraction. Circ Heart Fail. 2012; 5: 610-20.

28. Lumens J, Leenders GE, Cramer MJ, et al. Mechanistic evaluation of echocardiographic dyssynchrony indices: patient data combined with multiscale computer simulations. Circ Cardiovasc Imaging. 2012; 5: 491-9. 